\title{
Performance of routine SSFP cine cardiac MRI in the assessment of regurgitant valvular lesions, a comparison with echocardiography in a prospective cohort
}

\author{
Sabrina Sawhney, Jeannette McLaughlin, Kathy Halloran, Simcha Pollack, Jie J Cao \\ St Francis Hospital, Roslyn, State University of New York at Stony Brook, NY, USA
}

Correspondence: Jie Jane Cao. Address: St Francis Hospital, State University of New York at Stony Brook, 100 Port Washington Boulevard Roslyn, NY 11576, USA. Email: Jane.Cao@chsli.org

Received: March 4, 2016

Accepted: May 12, 2016

Online Published: May22, 2016

DOI : $10.5430 /$ ijdi.v3n2p37

URL: http://dx.doi.org/10.5430/ijdi.v3n2p37

\section{Abstract}

Background: The accuracy of valvular regurgitation assessment is uncertain by SSFP cine MR imaging. In this study we compared performance of cine images with Doppler echocardiography (echo) in a large prospective cohort.

Methods: We prospectively recruited 205 subjects who underwent MRI within 2 hours of echo. Regurgitation was assessed qualitatively and graded as none (0), trace (0.5), mild (1), mild to moderate (1.5), moderate (2.0), moderate to severe (2.5) and severe (3.0) by echo and by cine MRI. Grading from echo was used as reference.

Results: Prevalence of the mitral (MR), tricuspid (TR), aortic (AR) and pulmonic (PR) regurgitation was 79\%, 87\%, 29\% and $58 \%$ by echo, respectively. When regurgitation was absent the agreement between MRI and echo was good: $86 \%$, 79\%, 99\% and 96\% for MR, TR, AR and PR, respectively. However, few cases were detected by MRI for lesions $1+$ or less: $16 \%, 29 \%, 10 \%$ and $2 \%$, respectively. Detection of significant regurgitation ( $\geq 1.5+$ ) by MRI was reliable, $97 \%, 93 \%$, $90 \%$ and $100 \%$, with $25 \%, 14 \%, 30 \%$ and $33 \%$ underestimation of severity present, respectively while overestimation was rare.

Conclusion: SSFP cine MRI is reliable in detecting the absence or significant $(\geq 1.5+)$ regurgitant lesions but limited for mild or trivial diseases.

\section{Keywords}

Cardiovascular magnetic resonance imaging, Valvular regurgitation, Doppler echocardiography, Mitral regurgitation, Aortic regurgitation, Tricuspid regurgitation, Pulmonic regurgitation

\section{I ntroduction}

Cardiac Magnetic Resonance (CMR) Imaging has emerged as an important modality in the evaluation of patients with cardiomyopathy in whom valvular regurgitant abnormalities are highly prevalent. Traditionally, echocardiography (echo) has been the major imaging modality in assessing regurgitant valvular lesions which is subjected to operator variability in qualitative assessment. While phase contrast imaging of CMR is well validated in quantitative regurgitant assessment ${ }^{[1-12]}$ 
routine performance of phase contrast imaging adds to the scan time and breath-hold. Moreover, multiple concomitant valvular lesions commonly present in patients with cardiomyopathy complicate the quantitative assessment. Before the advent of balanced steady state free-precession (SSFP) sequence gradient echo sequence was the dominant sequence in cine imaging and was demonstrated to be effective in qualitative assessment of regurgitant lesions by assessing the signal void due to spin dephasing in moving protons ${ }^{[13-15]}$. While SSFP cine images is feasible in evaluating regurgitant valvular disease ${ }^{[13]}$ prospective study is lacking. Given the growing number of CMR in which SSFP cine is part of the routine protocol it can be clinically valuable if SSFP cine can provide qualitative evaluation of regurgitant lesion. In this study we compared diagnostic performance of SSFP cine MRI with Doppler echo in the qualitative assessment of regurgitant valvular diseases in a large prospectively recruited cohort.

\section{Material and methods}

The study subjects were pooled from two ongoing clinical research protocols which were approved by the Institution Review Board. Written Informed Consent was obtained from all participants. One protocol recruited subjects with cardiomyopathy of various etiologies including ischemic, dilated, infiltrative and hypertrophic cardiomyopathy. The second protocol recruited subjects with smoking history or with chronic obstructive pulmonary disease aged 20 to 85 years. Exclusions included claustrophobia; prosthetic valves or valvular apparatus; pacemaker/defibrillator implantation; or other metallic hazards. To test the performance of CMR on normal valves a small number $(\mathrm{N}=12)$ of normal volunteers were also included who had a normal ECG and echo. All participants underwent CMR within two hours of echo.

\subsection{Cardiac MRI}

CMR was performed with a Siemens 1.5 Tesla scanner (Siemens, Malvern, PA) using 8-element phased array surface coil. Cardiac structure and function were evaluated using balanced SSFP cine imaging in contiguous short axis slices and standard 2, 3, 4 chamber views, as well as longitudinal view of the right ventricular outflow tract. The images were acquired with retrospective ECG gating during breath-hold. The typical sequence specifications were as follows: field of view $240 \mathrm{~mm} \times 240 \mathrm{~mm}$, slice thickness of $8 \mathrm{~mm}$ with no interslice gap for short axis views or $6 \mathrm{~mm}$ thickness for longitudinal planes. TR (repetition time) $3.1 \mathrm{~ms}$, TE (echo time) 1.34, temporal resolution 30-40 ms, flip angle of $70^{\circ}$ and in-plane resolution of $1.3 \mathrm{~mm} \times 1.3 \mathrm{~mm}$. Left and right ventricular volume and function were assessed using commercially available software (QMass by Medis, Netherland).

\subsection{Echocardiography}

A transthoracic color Doppler echo was obtained by experienced technologists using a Philips IE33 echocardiograph (Andover, MA). Echo protocol was performed according to American Society of Echocardiography guidelines in standard views $^{[16]}$.

\subsection{Qualitative evaluation of regurgitant valvular lesions}

Valvular regurgitation scoring by CMR and Echo was performed with consensus reading by two independent experienced readers in their field. CMR and echo images were evaluated for the presence and extent of the regurgitant lesions following American Society of Echocardiography guidelines including visualization of the origin of the regurgitation jet and its width (vena contracta) and the spatial orientation of the regurgitant jet area in the receiving chamber ${ }^{[16]}$. The visual assessment of CMR followed echo guidelines taking into account of the regurgitant jet origin and width. Scoring scale for regurgitation was defined as: $0=$ no regurgitation; $0.5=$ trace regurgitation; $1.0=$ mild regurgitation; $1.5=$ mild to moderate regurgitation; $2.0=$ moderate regurgitation; 2.5 = moderate to severe regurgitation; $3.0=$ severe regurgitation. This scoring scale was used for both modalities (CMR and Echo). Due to low prevalence of stenotic disease (6 subjects with aortic stenosis), analyses were performed only to compare the regurgitant lesions. 


\section{Statistical analysis}

Continuous data is summarized by mean \pm standard deviation. Categorical data is presented as $\mathrm{N}$ (percent). Concordance data was obtained using Echo as the reference standard. CMR grading less than or equal to 0.5 of echo grading was considered concordant. $P$-values for the concordance data were generated by a nonparametric bootstrap resampling approach $^{[17]}$. For each of mitral (MR), tricuspid (TR), aortic (AR) and pulmonic (PR) regurgitation, the echo data was randomly permuted among the patients. These scores were subtracted from the original CMR score and the percentage of differences less than or equal to 0.5 was computed. This process was repeated 2,000 times to generate an empirical distribution of the differences under the null hypothesis of no association between the echo and CMR variables. A p-value was computed by comparing the actual percent of patients with echo and CMR pairs within 0.5 grading of each other to the bootstrapped distribution. CMR grading less than or equal to 1.0 of echo grading was defined as underestimation and greater than or equal to 1.0 of echo grading overestimation. The level of agreement between CMR and echo assessment was assessed using nonparametric statistics of Krippendorff's alpha index ${ }^{[18]}$. In addition, a weighted kappa with weights chosen to reflect the categories of agree/disagree was computed along with its $95 \%$ confidence interval. $P$-values less than .05 were deemed statistically significant. SAS 9.3 (SAS Institute, Inc, Cary, NC) was used for all statistical analyses.

\section{Results}

Of the 205 subjects included in the analysis the mean age was 56 years and $71 \%$ were male. Left ventricular dysfunction (ejection fraction $<50 \%$ ) was present in $77(38 \%)$ subjects. The details of the participants' characteristics are shown in Table 1 . The prevalence of MR, TR, AR and PR were $79 \%, 87 \%, 29 \%$ and $58 \%$ as detected by echo respectively.

Table 1. Characteristics of the participants $(\mathrm{N}=205)$

\begin{tabular}{ll}
\hline Variable & Mean \pm SD or N (\%) \\
\hline Age (years) & $56 \pm 14$ \\
Male & $146(71 \%)$ \\
Hypertension & $87(42 \%)$ \\
Diabetes millitus & $28(14 \%)$ \\
Dyslipidemia & $76(37 \%)$ \\
History of heart failure & $41(20 \%)$ \\
Body mass index $\left(\mathrm{kg} / \mathrm{m}^{2}\right)$ & $28 \pm 5$ \\
Heart rate $(\mathrm{bpm})$ & $66 \pm 14$ \\
Systolic blood pressure $(\mathrm{mmHg})$ & $132 \pm 21$ \\
Diastolic blood pressure (mmHg) & $75 \pm 13$ \\
Left ventricular end diastolic volume $\left(\mathrm{ml} / \mathrm{m}^{2}\right)$ & $86 \pm 27$ \\
Left ventricular end systolic volume $\left(\mathrm{ml} / \mathrm{m}^{2}\right)$ & $45 \pm 25$ \\
Left ventricular ejection fraction $(\%)$ & $50 \pm 12$ \\
Right ventricular end diastolic volume $\left(\mathrm{ml} / \mathrm{m}^{2}\right)$ & $73 \pm 21$ \\
RIght ventricular end systolic volume $\left(\mathrm{ml} / \mathrm{m}^{2}\right)$ & $35 \pm 11$ \\
Right ventricular ejection fraction $(\%)$ & $52 \pm 9$ \\
\hline
\end{tabular}

There was good agreement between CMR and echo when regurgitation was absent (see Table 1). However, CMR was limited in detecting trivial and mild $(\leq 1.0)$ regurgitant lesions (see Table 2). There were false positive findings by CMR where CMR detected trace or mild regurgitant (1+) lesions in $3 \%, 3 \%, 0.5 \%$ and $1.5 \%$ of mitral, tricuspid, aortic and pulmonic valve evaluations (see Table 3) when echo did not detect any regurgitant lesions. CMR detected the presence of regurgitation in more than $90 \%$ of the cases with significant regurgitation $(\geq 1.5+)$ (see Table 4$)$. Concordance with echo was found in more than $60 \%$ of those cases. Discrepancy was all due to underestimation by CMR and overestimation was absent. CMR Grading less than echo by 1.0 accounted for all cases with underestimation but 3 lesions (1 MR, 1 AR and 1 PR) in which underestimation was 1.5 grading less than echo. Examples of regurgitation lesions detected by echo and CMR are shown in Figures 1 through 6. 
Table 2. Agreement of SSFP cine MRI with echocardiography on mild or no regurgitant lesions

\begin{tabular}{lll}
\hline & Regurgitant severity & \\
\hline Lesion type & 0 & $0.5-1.0$ \\
Mitral regurgitation & $86 \%(36 / 44)$ & $16 \%(20 / 125)$ \\
Tricuspid regurgitation & $79 \%(23 / 29)$ & $29 \%(43 / 148)$ \\
Aortic regurgitation & $99 \%(143 / 144)$ & $10 \%(4 / 39)$ \\
Pulmonic regurgitation & $96 \%(82 / 85)$ & $2 \%(2 / 101)$ \\
\hline
\end{tabular}

Table 3. Number of false positive readings by MRI

\begin{tabular}{lllll}
\hline & \multicolumn{2}{l}{ Regurgitant severity } & & Total \\
\hline Lesion type & 0.5 & 1.0 & 1.5 & 6 \\
Mitral regurgitation & 2 & 3 & 1 & 6 \\
Tricuspid regurgitation & 2 & 4 & 0 & 1 \\
Aortic regurgitation & 0 & 1 & 0 & 3 \\
Pulmonic regurgitation & 0 & 3 & 0 & \\
\hline
\end{tabular}

Table 4. Performance of SSFP cine MRI on detecting significant regurgitant lesions $(\geq 1.5+)$

\begin{tabular}{llll}
\hline Lesion type & Overall detection & Concordant* $^{*}$ & Underestimation** \\
\hline Mitral regurgitation & $97 \%(25 / 36)$ & $69 \%(25 / 36)$ & $25 \%(9 / 36)$ \\
Tricuspid regurgitation & $93 \%(26 / 28)$ & $75 \%(21 / 28)$ & $14 \%(5 / 28)$ \\
Aortic regurgitation & $90 \%(18 / 20)$ & $60 \%(12 / 20)$ & $30 \%(6 / 20)$ \\
Pulmonic regurgitation & $100 \%(6 / 6)$ & $67 \%(4 / 6)$ & $33 \%(2 / 6)$ \\
\hline
\end{tabular}

* Lesion severity within 0.5 grading;

** Lesion severity by MRI was at less 1.0 grading or less than by echocardiography.
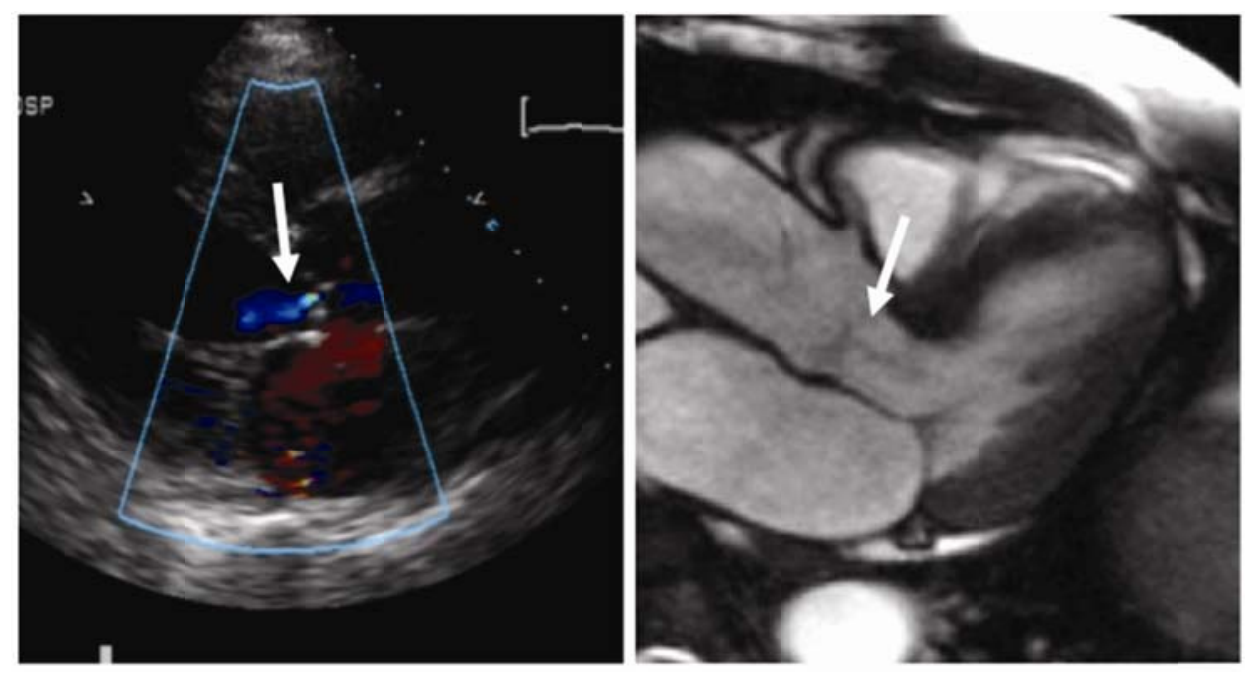

Figure 1. Mild aortic regurgitation by echocardiogram (left) in a parasternal long plane and by cardiac MRI (right) in 3-cahmber view

Overall, the concordance defined as CMR grading within 0.5 of echo grading was $78 \%, 78 \%, 88 \%$ and $85 \%$ for $\mathrm{MR}$, TR, AR and PR, respectively (all $p$ values $<.01$ ). Krippendorff's alpha index for ordinal data was $0.57,0.51,0.75$ and 0.17 and weighted kappa was $0.44,0.38,0.56$ and 0.17 for MR, TR, AR and PR respectively. 

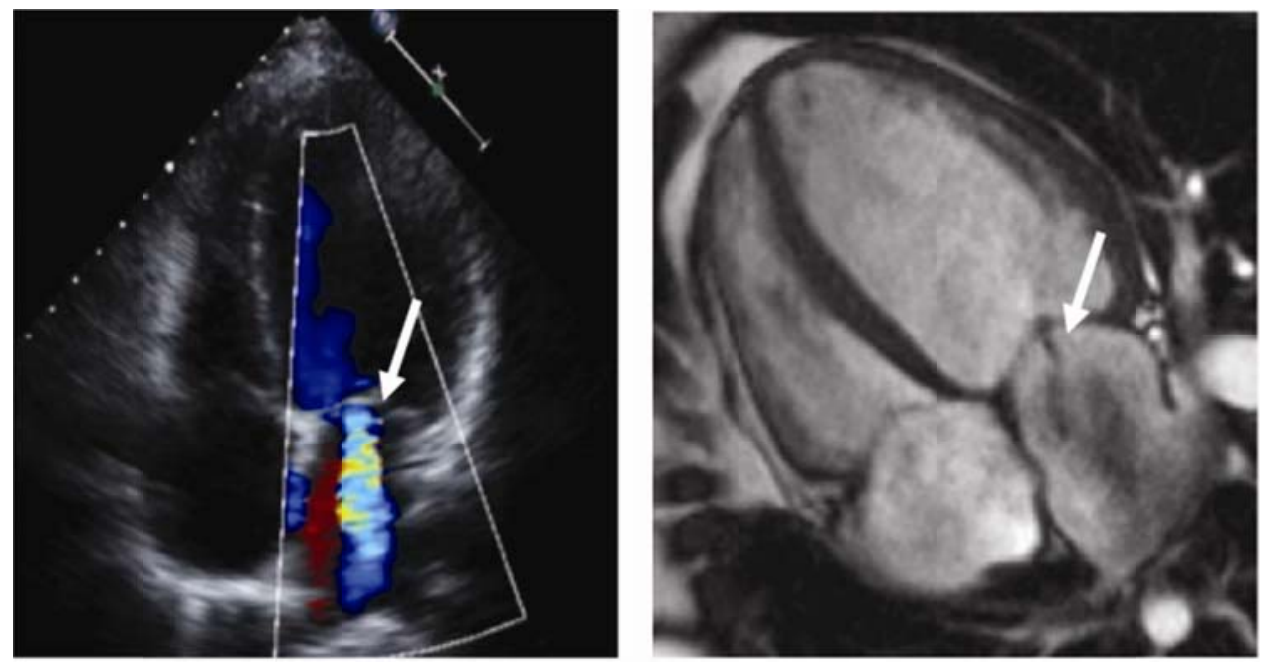

Figure 2. Moderate to severe mitral regurgitation by echocardiogram (left) and by cardiac MRI (right) in 4-chamber views
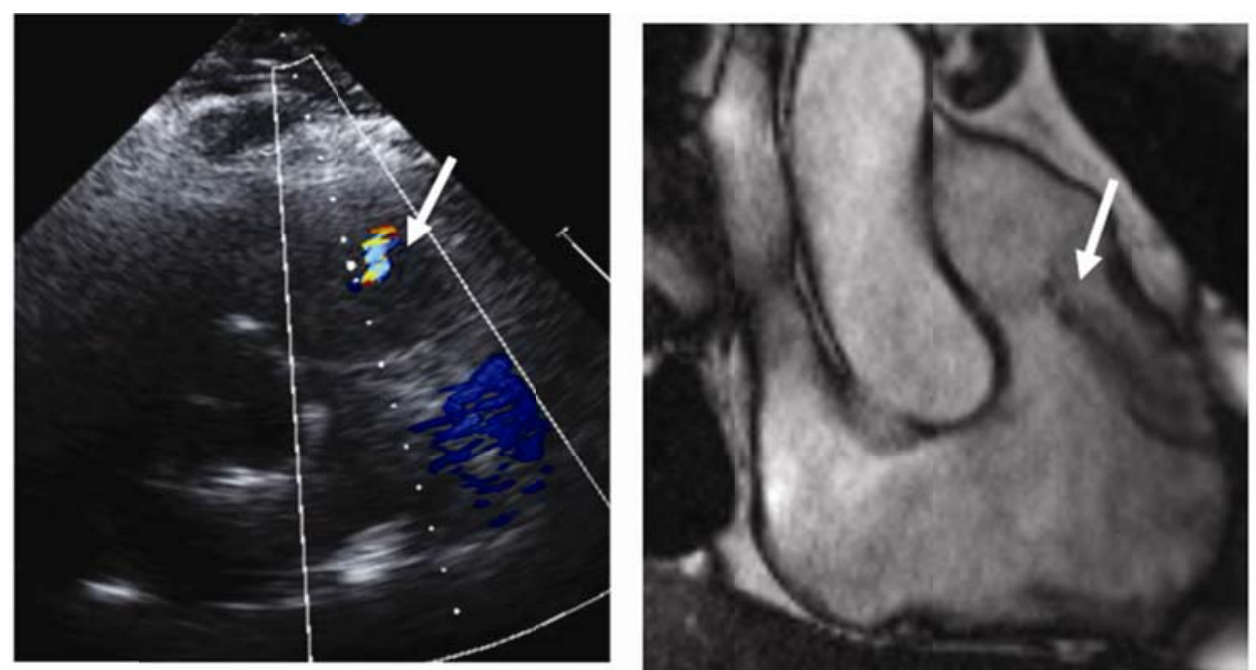

Figure 3. Mild to moderate pulmonic regugitation by echocardiogram (left) in parasternal short plane and by cardiac MRI (right) in right ventricular outflow tract view
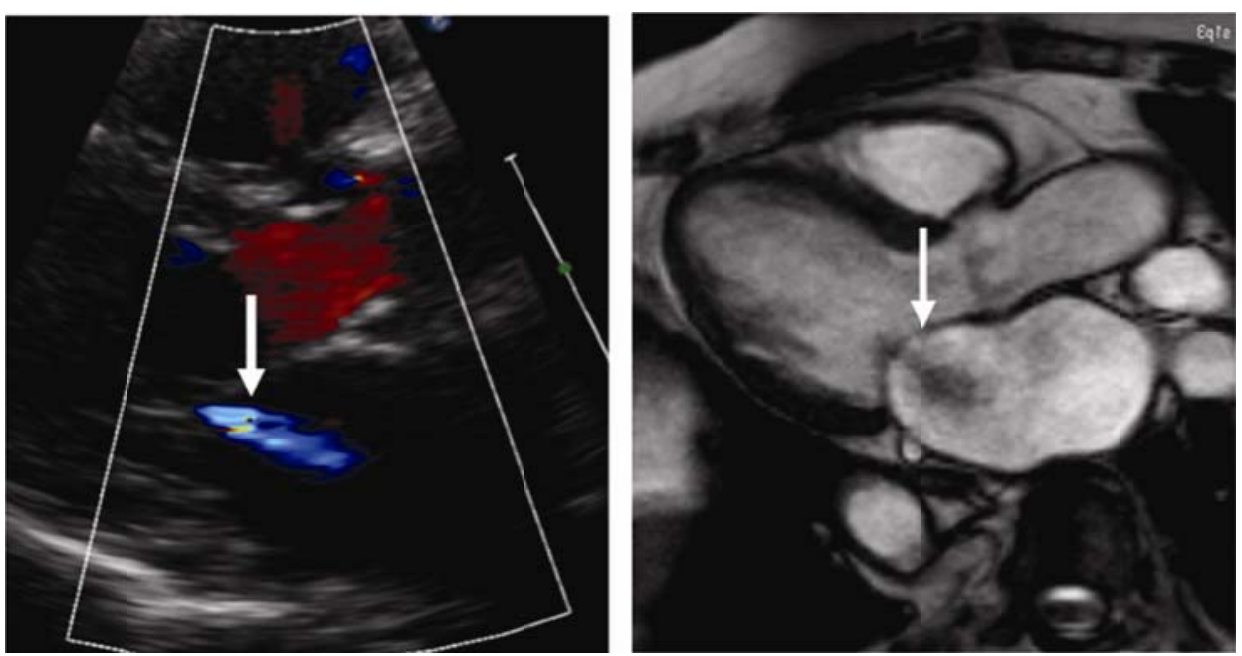

Figure 4. Mild mitral regurgitation by echocardiogram (left) in parasternal long plane and mild to moderate mitral regurgitation by cardiac MRI (right) in 3-chamber view 

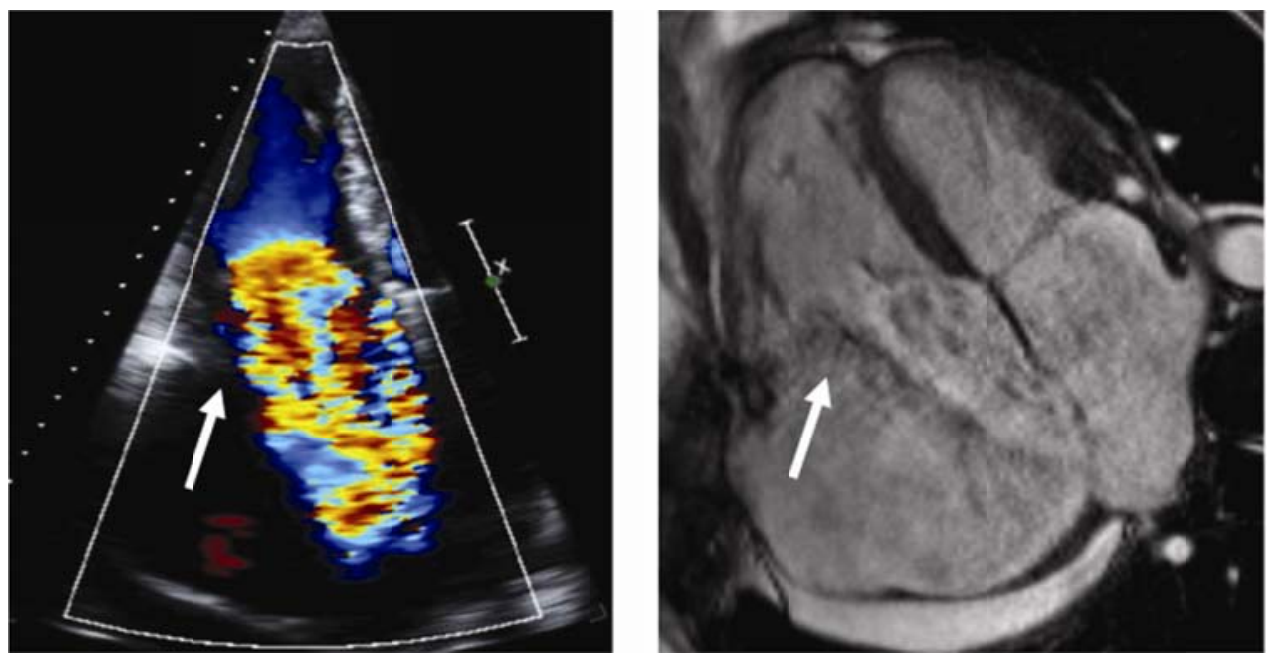

Figure 5. Severe tricuspid regurgitation by echocardiogram (left) and by cardiac MRI (right) in 4-chamber views
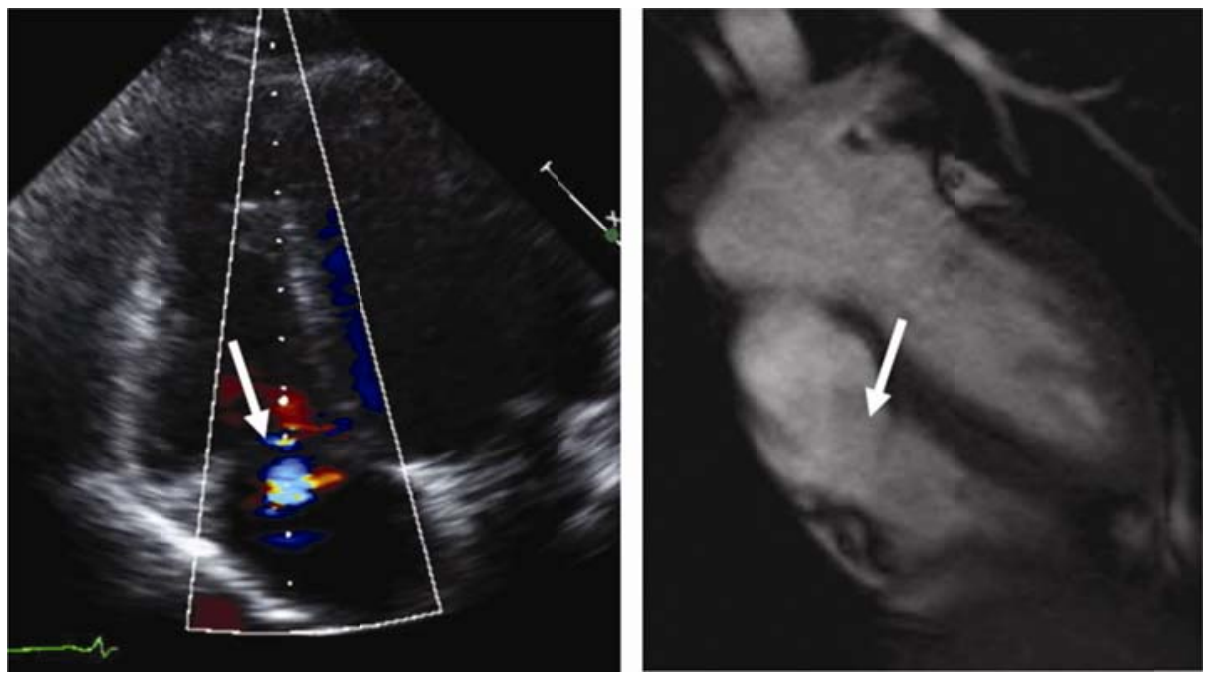

Figure 6. Mild tricuspid regurgitation by echocardiogram (left) and no significant regurgitation by cardiac MRI (right) in 4-chamber views

\section{Discussion}

In this study, we compared the diagnostic performance of SSFP cine MRI with Doppler echo in the qualitative assessment of valvular regurgitant lesions. We found that in more than three quarter of the cases (77\%-88\%), the regurgitation severity readings on CMR were within 0.5 grading score of the readings by echo for all four valves. CMR was reliable in detecting the absence or significant $(\geq 1.5+)$ regurgitant lesions but limited for mild or trivial diseases. The main source of discrepancy was underestimation of regurgitant severity by 1.0 grading by CMR assessment. Underestimation by $>1.0$ grading or overestimation was rare.

Regurgitant lesions are common in patients with cardiomyopathy. Evident in our series, up to $80 \%$ or more cases demonstrated some degree of MR or TR. As CMR becomes increasingly important in functional and tissue characterization for various cardiomyopathy regurgitant valvular lesions inevitably are the frequent findings in routine CMR studies. Phase contrast imaging alone or combined with volumetric assessment is well validated to quantitatively evaluate regurgitant lesions with high accuracy. However, phase contrast imaging is not without potential errors ${ }^{[19-21]}$. Furthermore it increases scan time and additional breath-holds which can be challenging in some patients if it is done 
systematically. Therefore, qualitative assessment of regurgitant lesions using routinely acquired cine images can be clinically valuable.

The assessment of regurgitant flow by cine imaging is made feasible through visualization of signal void due to spin dephasing in moving protons ${ }^{[22]}$. In the early CMR era gradient echo sequence with relatively long echo time was used in cine imaging which demonstrated excellent sensitivity and specificity in detecting regurgitant jet seen as signal void when compared to cineangiography or echo ${ }^{[13,14]}$. Gradient echo sequence has since been replaced by more rapid cine imaging method of balanced SSFP sequence. In SSFP images the flow related signal loss occurs in area where the voxels span a range of velocities. The signal voids are substantially related to the acceleration of blood rather than the velocity alone ${ }^{[2]}$. Due to shorter echo time in SSFP imaging there is reduced signal void from turbulent flow therefore lower sensitivity of detecting regurgitant lesions ${ }^{[13]}$ when compared to gradient echo sequence. In addition, the visual assessment is also subjected to slice positioning, partial volume effects and other sequence parameters. Despite broad clinical use of SSFP cine imaging, there is limited literature systematically evaluating its performance in the assessment of regurgitant lesions in a prospective cohort. Our findings suggest cine CMR was reliable when regurgitant lesions were absent. However, CMR appeared to have missed the majority of the cases with trace or mild regurgitation. It is important to recognize that CMR did not miss any significant lesion $(\geq 1.5)$ despite underestimation of severity was present. Small numbers of overestimation was only present in echo negative cases but none in cases with significant regurgitation. While cine CMR alone is clearly not adequate for patients referred for evaluation of regurgitant valvular disease it is valuable in estimating regurgitant severity with acceptable accuracy in more than three quarters of the patients. Small underestimation is relatively common and is the main source of discrepancy from echo evaluation.

We acknowledge the study limitations. While Doppler echo is the most commonly used modality for the evaluation of regurgitant valvular disease, the accuracy of assessment can be limited and subjected to operator variability when assessed qualitatively. The accuracy is enhanced when quantitative or semiquantitative measurements are incorporated. In this study we graded echo regurgitant severity based on qualitative reading alone which is a widely accepted practice in busy clinical setting. Hence the echo reference may not be set at its highest standard and is subject to reader's error. However this approach allows a fair comparison between CMR and echo using qualitative assessment only. Due to small number of patients with stenotic lesion we are unable to assess the accuracy of SSFP cine MRI in the qualitative evaluation of stenotic disease. The lack of comprehensive phase contrast imaging precludes the assessment of incremental accuracy of quantitative CMR evaluation. In the 4 available longitudinal views pulmonic or aortic valve is visualized only in one plane while mitral valve is seen in 3 views and tricuspid valve in 2 . Therefore the accuracy of valvular assessment may vary for each valve. We are also aware that valvular assessment by SSFP cine CMR is subject to image quality affected by arrhythmia, ECG gating, real time imaging, artifact from prosthetic valvular apparatus and patient factors such as poor breath-hold and motions. Findings observed in $1.5 \mathrm{~T}$ scanner may not be generalized in higher field magnetic environment.

In conclusion using routine SSFP cine image alone in long axis views CMR can accurately detect regurgitant lesions in mitral, tricuspid, aortic and pulmonic valves in more than three quarters of cases. Cine CMR is reliable in assessing cases with absent or significant regurgitant diseases. Relatively small underestimation is the main cause of discrepancy from echo reading. Our findings suggest that cine CMR can be valuable in routine clinical scan in estimating regurgitant lesions among patients with prevalent valvular diseases.

\section{Funding source}

The study is funded by St Francis Research Foundation.

\section{References}

[1] Kilner PJ, Gatehouse PD, Firmin DN. Flow measurement by magnetic resonance: a unique asset worth optimising. J Cardiovasc Magn Reson. 2007; 9: 723-728. PMid:17613655 http://dx.doi.org/10.1080/10976640701465090 
[2] Myerson SG. Heart valve disease: investigation by cardiovascular magnetic resonance. J Cardiovasc Magn Reson. 2012 ; 14 : 7. PMid:22260363 http://dx.doi.org/10.1186/1532-429X-14-7

[3] Globits S, Frank H, Mayr H, et al. Quantitative assessment of aortic regurgitation by magnetic resonance imaging. Eur Heart J. 1992; 13: 78-83. PMid:1577036

[4] Dulce MC, Mostbeck GH, O'Sullivan M, et al. Severity of aortic regurgitation: interstudy reproducibility of measurements with velocity-encoded cine MR imaging. Radiology. 1992; 185: 235-240. PMid:1523315 http://dx.doi.org/10.1148/radiology.185.1.1523315

[5] Honda N, Machida K, Hashimoto M, et al. Aortic regurgitation: quantitation with MR imaging velocity mapping. Radiology. 1993; 186: 189-194. PMid:8416562 http://dx.doi.org/10.1148/radiology.186.1.8416562

[6] Kutty S, Whitehead KK, Natarajan S, et al. Qualitative echocardiographic assessment of aortic valve regurgitation with quantitative cardiac magnetic resonance: a comparative study. Pediatr Cardiol. 2009; 30: 971-977. PMid:19636486 http://dx.doi.org/10.1007/s00246-009-9490-6

[7] Stork A, Franzen O, Ruschewski H, et al. Assessment of functional anatomy of the mitral valve in patients with mitral regurgitation with cine magnetic resonance imaging: comparison with transesophageal echocardiography and surgical results. Eur Radiol. 2007; 17: 3189-3198. PMid:17549489 http://dx.doi.org/10.1007/s00330-007-0671-5

[8] Fujita N, Chazouilleres AF, Hartiala JJ, et al. Quantification of mitral regurgitation by velocity-encoded cine nuclear magnetic resonance imaging. J Am Coll Cardiol. 1994; 23: 951-958. http://dx.doi.org/10.1016/0735-1097(94)90642-4

[9] Chan KM, Wage R, Symmonds K, et al. Towards comprehensive assessment of mitral regurgitation using cardiovascular magnetic resonance. J Cardiovasc Magn Reson. 2008; 10: 61. PMid:19102740

[10] Rebergen SA, Chin JG, Ottenkamp J, et al. Pulmonary regurgitation in the late postoperative follow-up of tetralogy of Fallot. Volumetric quantitation by nuclear magnetic resonance velocity mapping. Circulation. 1993; 88(5 Pt 1): 2257-2266. PMid:8222120

[11] Vliegen HW, van SA, de Roos A, et al. Magnetic resonance imaging to assess the hemodynamic effects of pulmonary valve replacement in adults late after repair of tetralogy of fallot. Circulation. 2002; 106: 1703-1707. PMid:12270866 http://dx.doi.org/10.1161/01.CIR.0000030995.59403.F8

[12] Li W, Davlouros PA, Kilner PJ, et al. Doppler-echocardiographic assessment of pulmonary regurgitation in adults with repaired tetralogy of Fallot: comparison with cardiovascular magnetic resonance imaging. Am Heart J. 2004; 147: 165-172. http://dx.doi.org/10.1016/S0002-8703(03)00527-1

[13] Krombach GA, Kühl H, Bücker A, et al. Cine MR imaging of heart valve dysfunction with segmented true fast imaging with steady state free precession. J Magn Reson Imaging. 2004; 19: 59-67. PMid:14696221 http://dx.doi.org/10.1002/jmri.10428

[14] Wagner S, Auffermann W, Buser P, et al. Diagnostic accuracy and estimation of the severity of valvular regurgitation from the signal void on cine magnetic resonance images. Am Heart J. 1989; 118: 760-767. http://dx.doi.org/10.1016/0002-8703(89)90590-5

[15] Suzuki J, Caputo GR, Kondo C, et al. Cine MR imaging of valvular heart disease: display and imaging parameters affect the size of the signal void caused by valvular regurgitation. Am J Roentgenol. 1990; 155: 723-727. PMid:2119099 http://dx.doi.org/10.2214/ajr.155.4.2119099

[16] Zoghbi WA, Enriquez-Sarano M, Foster E, et al. Recommendations for evaluation of the severity of native valvular regurgitation with two-dimensional and Doppler echocardiography. J Am Soc Echocardiogr. 2003; 16: 777-802. http://dx.doi.org/10.1016/S0894-7317(03)00335-3

[17] Efron B, Tibshirani R. An Introduction to the Bootstrap. Monographs on Statistics and Applied Probability, No. 57. London: Chapman \& Hall Press. 1993. http://dx.doi.org/10.1007/978-1-4899-4541-9

[18] Krippendorff K. Estimating the reliability, systematic error, and random error of interval data. Educational and Psychological Measurement. 1970; 30: 61-70. http://dx.doi.org/10.1177/001316447003000105

[19] Chernobelsky A, Shubayev O, Comeau CR, et al. Baseline correction of phase contrast images improves quantification of blood flow in the great vessels. J Cardiovasc Magn Reson. 2007; 9: 681-685. PMid:17578724 http://dx.doi.org/10.1080/10976640601187588

[20] Miller TA, Landes AB, Moran AM. Improved accuracy in flow mapping of congenital heart disease using stationary phantom technique. J Cardiovasc Magn Reson. 2009; 11: 52. PMid:20003318 http://dx.doi.org/10.1186/1532-429X-11-52

[21] Gatehouse PD, Rolf MP, Graves MJ, et al. Flow measurement by cardiovascular magnetic resonance: a multi-centre multi-vendor study of background phase offset errors that can compromise the accuracy of derived regurgitant or shunt flow measurements. $\mathrm{J}$ Cardiovasc Magn Reson. 2010; 12: 1-8. PMid:20074359 http://dx.doi.org/10.1186/1532-429X-12-5

[22] Evans AJ, Blinder RA, Herfkens RJ, et al. Effects of turbulence on signal intensity in gradient echo images. Invest Radiol. 1988; 23: 512-518. PMid:3170139 http://dx.doi.org/10.1097/00004424-198807000-00006 